\title{
Day length-dependent thermal COP1 dynamics integrate conflicting seasonal cues in the control of Arabidopsis elongation
}

\section{AUTHORS}

Cristina Nieto ${ }^{1,2 .^{*}}$, Pablo Catalán ${ }^{3,4^{*}}$, Luis Miguel Luengo ${ }^{1,5}$, Martina Legris ${ }^{6}$, Vadir López-Salmerón ${ }^{1}$, Jean Michel Davière ${ }^{1}$, Jorge Casal ${ }^{6,7}$, Saúl Ares ${ }^{3,8+}$ and Salomé Prat ${ }^{1,5+}$

1 Plant Molecular Genetics Department, CNB-CSIC, Darwin 3, 28049 Madrid, Spain

2 Plant Genetic Resources Centre (INIA-CSIC) Autovía A2, km 32, 28805 Alcalá de Henares, Madrid, Spain

${ }^{3}$ Mathematics Department, Universidad Carlos III de Madrid, and Interdisciplinary Group of Complex Systems (GISC), Avenida de la Universidad 30, 28911 Leganés, Madrid, Spain

${ }^{4}$ Biosciences, University of Exeter, Exeter EX4 4QD, UK

${ }^{5}$ Plant Development and Signal Transduction, Centre for Research in Agricultural Genomics (CRAG), 08193 Cerdanyola Barcelona, Spain

${ }^{6}$ Fundación Instituto Leloir, Instituto de Investigaciones Bioquímicas de Buenos Aires,

Consejo Nacional de Investigaciones Científicas y Técnicas, 1405 Buenos Aires, Argentina

7 Instituto de Investigaciones Fisiológicas y Ecológicas Vinculadas a la Agricultura, Facultad de Agronomía, Universidad de Buenos Aires, Consejo Nacional de Investigaciones Científicas y Técnicas, 1417 Buenos Aires, Argentina ${ }^{8}$ Systems Biology Department, CNB-CSIC, Darwin 3, 28049 Madrid, Spain

*These two authors contributed equally to this work +Co-corresponding authors: $\underline{\text { saul.ares@csic.es, salome.prat@cragenomica.es }}$ 


\section{ABSTRACT}

As the summer approaches, plants experience enhanced light inputs and elevated temperatures, two environmental cues with an opposite morphogenic impact. How plants integrate this conflicting information throughout seasons remains unclear. Key components of the plant response to light and temperature include phytochrome $B$ (phyB), PHYTOCROME INTERACTING FACTOR 4 (PIF4), EARLY FLOWERING 3 (ELF3) and CONSTITUTIVE PHOTOMORPHOGENIC 1 (COP1). Here, we used hypocotyl lengths of single and double mutant/over-expression lines to fit a mathematical model incorporating known interactions of these genes. The fitted model recapitulates day length-dependent thermoelongation of all lines studied, and correctly predicts temperature responsiveness of new genotypes. Whilst previous works pointed to a lightindependent thermal function of COP1, simulations of our model suggested that COP1 has a role in temperature-signaling only during daytime. Based on by this prediction, we show that COP1 overexpression increases thermal response in continuous white light, while it has little effect in darkness. Defective thermal response of cop1-4 mutants is epistatic to phyB-9 and elf3-8, indicating that COP1 activity is essential to the transduction of phyB and ELF3 thermosensory function. Our model accurately captures phyB, ELF3 and PIF4 dynamics, providing an excellent toolbox for identification of best allelic combinations towards optimized crops resilience to climate change at different geographical latitudes. 


\section{INTRODUCTION}

Light and temperature are key environmental factors that shape plant growth patterns according to the prevailing conditions of the environment. Growth of the hypocotyl, for instance, proceeds very rapidly in young Arabidopsis seedlings buried in the soil, to speed up exposure of photosynthetic organs to sunlight. Upon emergence, light severely reduces the hypocotyl growth rate, while this is reassumed on seedlings exposure to neighboring vegetation that threat resources availability. Warm temperatures alike enhance hypocotyl growth to facilitate cooling of aerial tissues. The choice of sowing date is a critical decision in crop management to optimize overlap with the most favorable season. As sowing dates progress towards the warmer season, plants sense the increased light input of longer day lengths. However, how contrasting effects of light and temperature integrate to the control of thermomorphogenic development across seasons remains poorly understood.

The predominant photoreceptors inhibiting hypocotyl growth in Arabidopsis are phytochrome B (phyB), phyA, and cryptochromes. phyB acts as a main thermosensor, featuring a cross-talk interaction of light and temperature information already at the sensor level. phyB is synthetized in an inactive form ( $\mathrm{Pr})$ and photo-converted upon redlight $(R)$ perception into the active Pfr state that is translocated into the nucleus for light signaling transduction ${ }^{1}$. Far red-light (FR) rapidly reverses $\mathrm{Pfr}$ into the $\mathrm{Pr}$ state and through this conversion phyB senses changes in the R/FR ratio that result from neighboring plants ${ }^{2,3}$. Moreover, phyB Pfr slowly returns into the inactive Pr form, in a process termed thermal or dark reversion that is accelerated by warm temperatures ${ }^{4,5}$. While phyA and cryptochromes have a main role in light-induced transcriptional reprogramming, so far there is no evidence for these photoreceptors acting as temperature sensors.

ELF3 confers the circadian clock's "evening complex" (EC) thermal responsiveness via a prion-like thermosensor domain that reversibly directs protein phase transition ${ }^{6}$. ELF3, 
ELF4 and LUX occupancy of their target loci is strongly reduced at warmer temperatures ${ }^{7,8}$, with loss of elf3, elf4, or lux function leading to increased growth at $22^{\circ} \mathrm{C}$, indicative of a constitutively activated thermal response in these mutants ${ }^{9}$. Temperatureinduced elongation is arbitrated by transcriptional activation of cell wall loosening and auxin biosynthetic/ signaling genes by the PIF4 and PIF7 factors ${ }^{10,11}$. Translation of PIF7 is increased at warm temperatures through structural changes of an RNA hairpin loop within the $5^{\prime}$ untranslated transcript region, with this control likely having a prevalent role on daytime activation of the thermomorphogenic pathway under longer day lengths.

Light activated phyB suppresses PIF4 activity by signaling its phosphorylation and subsequent degradation by the $26 \mathrm{~S}$ proteasome, in addition to prevent PIF4 binding to its target promoters ${ }^{12,13}$. The circadian clock regulates rhythmic PIF4 expression via transcriptional repression by the LUX, ELF4 and ELF3 proteins, comprising the core evening complex loop ${ }^{14}$. The EC is recruited by the LUX factor to the PIF4 promoter, suppressing PIF4 expression during early night ${ }^{15}$.

Additionally, phyB Pfr inactivates the E3 ligase COP1, which acts as a photomorphogenesis suppressor in the dark. COP1 localizes into the nucleus in darkness, where it targets degradation of multiple photomorphogenesis-promoting factors, including LONG HYPOCOTYL IN FAR-RED 1 (HFR1) and ELONGATED HYPOCOTYL (HY5) ${ }^{16-18}$. Red-light dependent phyB and SPA1 interaction dissociates the SPA1-COP1 complex and suppresses COP1 activity ${ }^{19}$, besides triggering COP1 nuclear exclusion through a yet elusive mechanism ${ }^{20}$. HFR1 and HY5 promote photomorphogenesis by antagonizing PIF4, through formation of inactive HFR1-PIF4 complexes and competitive interaction of HY5 with the PIF4 cognate elements ${ }^{21-24 .}$ Elevated temperatures promote in turn COP1 nuclear accumulation ${ }^{25}$, hence alleviating HY5 suppressive effects. COP1 is also reported to trigger ELF3 destabilization during ELF3-dependent recruitment of GIGANTEA (GI) for COP1 degradation ${ }^{26}$, although relevance of this process in growth responses remains unknown. 
Together, these observations reveal that phyB, ELF3, PIF4 and COP1 form a thermal network where all of these regulators physically and functionally interact with each other. Along with phyB suppression of PIFs and COP1 activity, COP1 is shown to facilitate phyB Pfr turnover under extended light ${ }^{27}$. Furthermore, phyB favors ELF3 accumulation presumably by suppressing protein destabilization by COP $1{ }^{28}$, besides co-occupying at cooler temperatures many of the EC target loci 7 . PIFs, in turn, promote COP1-mediated phyB degradation ${ }^{27}$, and recruit phyB into the Light-Response BTB1 and 2 (LRB1 and LRB2) complex, involved at phyB and PIFs ubiquitination and mutual destruction 29,30 Thus, extensive connectivity of these regulators makes them prime candidates for modeling studies, to dissect their exact roles in thermal elongation and assess whether their thermosensory function is dependent on light.

Here we investigated the output of this network in seedlings grown under different day lengths and $22^{\circ} \mathrm{C} / 28^{\circ} \mathrm{C}$, as a mimic of seasonal information. We used the hypocotyl lengths of mutants and overexpresser lines to fit a mathematical model based on the molecular interactions of these regulators. We validate the predicted molecular dynamics of these components and use the generated model to weigh their relative contribution to thermal elongation as influenced by day length.

\section{RESULTS}

\section{Day length-dependent roles of phyB, ELF3, and COP1 in thermal elongation}

To gain a better understanding on the combined roles of phyB, ELF3, and COP1 in coordinating thermomorphogenesis (Fig.1A), we examined temperature-induced elongation of single and double mutants and overexpression lines in the Col-0 background. Light effects on thermal responsiveness were analyzed by measuring their hypocotyl lengths under continuous dark, continuous white light (CWL), and 8h, 12h and $16 \mathrm{~h}$ light cycles, at a temperature of $22^{\circ} \mathrm{C}$ or $28^{\circ} \mathrm{C}$ (Fig.1A, Material and Methods). 
Thermal response of wild-type plants was found in these studies to be inversely correlated with day length. Hypocotyl elongation was greatest at $8 \mathrm{~h}$ light and progressively reduced by $12 \mathrm{~h}$ and $16 \mathrm{~h}$ day lengths, while Col-0 seedlings lacked any thermal response in CWL (Fig.1A). Thermal elongation was also significantly reduced in continuous darkness, suggesting that thermal growth requires of initial phyB Pfr photoconversion and photomorphogenic induction, in addition to alternating light/dark cycles. This is consistent with gating by the circadian clock to be pivotal to this response. Thermal growth was moreover suppressed in pif4 and pifq mutants (Fig.1A), while it was notably enhanced in phyB-9 mutant and PIF4ox lines, in agreement with phyB acting at temperature perception ${ }^{4}$ and a downstream role of PIF4 in promoting thermal elongation ${ }^{10}$. However, as opposed to the inhibitory effects of increasing light hours in the wild-type and pif mutants, longer day lengths enhanced thermal responsiveness of the phyB-9 and PIF4ox lines. These plants showed almost maximal thermoelongation in CWL, consistently with temperature inactivating phyB Pfr also during daytime 5 .

Thermal response was impaired in all cop1-4 genotypes, demonstrating that COP1 activity is essential to thermal responsiveness. These plants are much shorter in darkness than pif4 and pifq mutants, in line with the strong constitutive photomorphogenic phenotype of this weak allele. Thermal sensitivity defects were also more severe than for pif4 and pifq mutants, with cop1-4 seedlings failing to elongate at warmer temperatures, whereas pif4 mutants elongated in shorter day lengths. More importantly, COP1 over-expression caused a similarly enhanced thermoresponse in CWL as seen in phyB-9 mutants and PIF4ox lines, indicating that COP1 turnover of PIFsantagonizing factors like $\mathrm{HY} 5$ is pivotal to thermomorphogenic growth. A greater thermal response in CWL was also observed in the hy5 mutant (Supp. Fig. 1A), consistent with targeted HY5 turnover being to a large extent responsible of this phenotype ${ }^{31}$. COP1- 
$O E$ lines were also more elongated in CWL than the phyB-9 mutant, suggesting that COP1-dependent PIFs stabilization ${ }^{32,33}$ is biologically more relevant in prolonged light. Alternatively, other phytochromes may suppress phyB-9 elongation.

Although PIF4 was reported to play a prominent role in driving thermomorphogenesis, pif4 mutants showed a wild-type thermal response in darkness or shorter day lengths (Tukey's HSD test, Fig.1A), presumably due to temperature responsive PIF7 activity ${ }^{11}$. Thermal sensitivity was however decreased in pifq mutants, indicating that PIF1, PIF3 and PIF5 contribute as well to thermal growth. Hypersensitive response to day length of $P H Y B o x$ lines is indeed associated with suppressed thermomorphogenesis, indicating that thermal phyB Pfr inactivation leads to stabilization of all PIFs. Furthermore, PHYBox lines show remarkable thermoelongation in darkness, underscoring a light independent function of other thermosensors.

As earlier reported, elf3-8 mutants showed de-repressed thermal elongation at $22^{\circ} \mathrm{C}{ }^{9}$, though a residual response was still observed in all tested conditions (Tukey's HSD test, Fig.1A). Sensitivity to day length was the most significant among all assayed genotypes but it did not change with temperature, hence indicating that gating effects of the clock are important to mediate suppression of thermal responsiveness in longer day lengths. Unlike PIF4ox lines, growth of elf3-8 mutants was inhibited in CWL, suggesting that ELF3 has other functions than gating PIF4 expression ${ }^{15}$. In this regard, ELF3ox lines were impaired in thermal elongation in long days ( $16 \mathrm{~h}$ light), though they behaved as the wild type in other diel conditions. These plants were as tall in darkness as elf3-8 mutants, showing that interaction with phyB Pfr is key to ELF3 function ${ }^{34}$.

ELF3ox cop1-4 and elf3-8 cop1-4 lines exhibited an identical photomorphogenic development and defective thermal elongation as cop1-4 mutants. ELF3ox cop1-4 lines were somehow shorter than cop1-4 in darkness, in contrast to the tall hypocotyls of etiolated ELF3ox (Fig.1A). This phenotype suggests that COP1 antagonizes ELF3 
presumably by targeting degradation of the protein, whereas light may partially suppress ELF3 turnover.

Together, these data reveal that phyB, COP1 and ELF3 govern thermal elongation under diel conditions in a highly intertwined fashion (Fig. 1B). Dynamic changes in phyB Pfr levels as a result of external light and temperature cues drive thermal growth by suppressing PIFs nuclear accumulation, along with COP1-mediated turnover of HY5, and presumably ELF3. On the other side, thermal elongation of phyB-9 and $P H Y B o x$ lines in the dark reveals that ELF3 and possibly COP1 have a role in modulating thermal responsiveness, independently of phyB Pfr.

\section{A mathematical model on activity of these regulators captures thermal growth of all genotypes}

To assess biological significance of these regulatory events, measured hypocotyl lengths were used to fit the parameters of a mathematical model built on the known interactions of these regulators (Fig. 1B).

We used four differential equations (Supplementary Material, Eq. (S1)) that captured active levels of phyB, PIFs, ELF3 and COP1, and a fifth one that represented hypocotyl growth. For phyB dynamics we adhered to the thermal reversion model previously described ${ }^{4}$, where phyB is photoactivated in the light but spontaneously reversed to its inactive $\operatorname{Pr}$ form in the dark, whereas the rate of phyB inactivation is temperature dependent. Dynamics of ELF3 was modelled based on its circadian control, with ELF3 transcripts following a broad peak of expression at dusk ${ }^{15,35}$. COP1 was modeled in quite an agnostic way as its nuclear levels depend on interactions with several photoreceptors for which we had no experimental information ${ }^{36}$.

PIFs activity was modelled according to ELF3 repressing PIF4 and PIF5 transcription, with two additional terms incorporating phyB inhibition of PIFs activity, and COP1dependent stabilization of the PIF proteins ${ }^{30,32}$. Finally, hypocotyl growth was 
represented as the PIFs-dependent activation of growth-related genes ${ }^{37}$, and the competitive effects of ELF3, phyB, and HY5, on PIFs occupancy/transcriptional activation of these genes 1 . No additional equation was included for HY5, to which its steady state levels were assumed to mainly depend on proteolytic degradation by COP1 39. A full mathematical definition of the model is provided in Supplementary Material.

A custom simulated annealing algorithm (Supplementary Material) was used to fit these different equations to the growth dataset generated for the various genotypes (Methods). The fit showed that several parameters of the initial full model, Eq. (S3), were consistent with values of zero, so we proceeded to simplify the equations by eliminating these terms, Eq. (S1). In particular, the model worked equally well excluding the effect of COP1 on phyB degradation and PIFs' action on phyB desensitization ${ }^{27}$. This does not mean that these interactions do not exist, but rather that they are quantitatively less relevant than others in our experimental conditions, or that their effect is captured by other terms. The final model reproduced the dominant trends of all genetic backgrounds (Fig. 1A, solid lines), demonstrating that dynamic control of these regulators is sufficient to explain their thermomorphogenic phenotypes. Moreover, it allowed extrapolating their thermal behavior in other day length conditions (Supp. Fig. 1A), in addition to correctly predict thermal responsiveness of new genotypes (Supp. Fig. 1B). The model predicts, for instance, that suppressed thermal responsiveness of $P H Y B o x$ lines is partly restored under $4 \mathrm{~h}$ light. Validation of this behavior (Supp. Fig. 1B) therefore shows that thermal Pfr inactivation reverses growth inhibition by excessive Pfr conversion in the light. The model also estimates that thermal responsiveness of double phyB-9 elf3-8 mutants linearly increases with day length, as seen in phyB-9. Experimental verification of this phenotype supports an additive effect of ELF3 and phyB on thermomorphogenesis (Supp. Fig. 1A). We also validated the cop 1-4 alike phenotype of phyB-9 cop1-4 mutants, showing that enhanced COP1 action driven by Pfr thermal inactivation is key to 
thermomorphogenic growth (Supp. Fig. 1A). All code and data used for fitting and model simulations can be found at https://github.com/pablocatalan/hypocotyl.

\section{Temperature-dependent dynamics of ELF3 and PIF4}

ELF3 is a key node required for transmitting temperature information to the regulated gating of PIF4 and PIF5 expression at night ${ }^{40}$. We therefore used the luciferase reporter pELF3::LUC, pELF3::ELF3-LUC, pPIF4::LUC and pPIF4::PIF4-LUC lines for the noninvasive imaging of temperature effects on ELF3 and PIF4 peak expression and protein levels. Lines expressing the ELF3-LUC and PIF4-LUC fusions showed identical protein

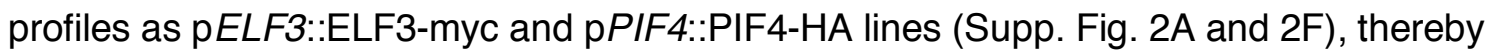
allowing the analysis of rhythmic changes in these proteins during several consecutive days (Fig. 2A and 2B; Methods).

Consistent with previous reports ${ }^{15,35}, \quad$ pELF3::LUC expression showed a robust circadian rhythm peaking during nighttime (Supp. Fig. 2B and 2D). A roughly equivalent pattern was observed for the ELF3-LUC protein, except by showing some accumulation in short days in the light (Fig. 2A). Warm temperatures dampened $\mathrm{p} E L F 3$ activity and slightly advanced its oscillation phase. Moreover, $\mathrm{p} E L F 3$ waveform switched in short days to a steady state pattern, whereby LUC activity attained a constant plateau on dark transition and returned to basal levels by end of night (Supp. Fig. 2B). Interestingly, reduction of ELF3-LUC activity was lesser than for $\mathrm{pELF3}$ transcription, indicating that ELF3 accumulates at warmer temperatures (Fig. 2A). ELF3-LUC bioluminescence was higher at $28^{\circ} \mathrm{C}$ during the day, and did not decline on dark transition as observed at $22^{\circ} \mathrm{C}$. The same trend is seen in western blots conducted with pELF3::ELF3-myc lines (Supp. Fig. 2A), implying that temperature inactivates the EC via other molecular mechanisms than the direct control of ELF3 protein stability.

In short days, pPIF4 expression increased before dawn, as earlier described ${ }^{15}$. Its transcription peaked during daytime and rapidly declined at night (Supp. Fig. 2G). Consistently with temperature suppressing gating by the EC ${ }^{40}$, LUC levels were elevated 
at $28^{\circ} \mathrm{C}$ earlier at night (Supp. Fig. 2G). In line with previous reports ${ }^{41}, p$ PIF4 was however expressed in long days exclusively during daytime (Supp. Fig. 2l). Also, temperature did not lead to precocious pPIF4 up-regulation as in short days (Supp. Fig. 2I), but enhanced the amplitude and delayed pPIF4 phase. This suggests that the EC gates PIF4 expression during long day afternoon, as opposed to its nighttime repressive action in short days (Supp. Fig. 2G and 2J).

PIF4-LUC levels had a similar profile as pPIF4 expression. The protein was in short days stabilized by end of the night (Fig. 2B), while in long days it decayed earlier than pPIF4 expression during late afternoon (Supp. Fig. 2H). Also, premature decay in PIF4-LUC levels is lost at $28^{\circ} \mathrm{C}$, where PIF4-LUC and pPIF4 activities essentially overlap (Fig. 2E). Thus, PIF4 protein stabilization during long days late afternoon/ early night may provide a window for thermal elongation, in the absence of elevated PIF4 expression at night. Remarkably, although we implemented ELF3 activity as oscillating between two stable states (Supplementary Material), the model captures the ELF3 protein dynamics we observed experimentally (Fig. 2A, 2D and Supp. Fig. 2E). Total ELF3-LUC levels were greater in the day at $28^{\circ} \mathrm{C}$, whereas differences in protein levels diminished during nighttime, consistent with the model's prediction. Likewise, the model predicts PIF4 activity to be greater at night, and significantly enhanced at $28^{\circ} \mathrm{C}$ (Fig. 2E). PIF4-LUC abundance in fact increases at $28^{\circ} \mathrm{C}$ during nighttime or late afternoon (Fig. $2 \mathrm{~B}$ and Supp. Fig. $2 F$ ), although it is also elevated in the day as seen in previous reports ${ }^{41-43}$. This PIF4 pool is admitted to be mostly inactive due to inhibition by phyB, HY5, or the DELLA repressors (Park et al., 2017; Johansson et al., 2014; de Lucas et al., 2008), the model thereby correctly estimating active PIF4 levels.

\section{Diurnal control of phyB thermal reversion}

Reduced steady-state phyB Pfr levels at warmer temperatures lead to the disassembly of large phyB photobodies, thought to be active sites for phyB signaling ${ }^{5,45}$. Formation of these subnuclear domains in bright light tightly correlates with inhibition of hypocotyl 
growth ${ }^{46}$, whereas increased Pr/Pfr ratios, in the shade or dim light, give rise to phyB redistribution into many small foci and the nucleoplasm, thereby providing an excellent cell biology readout for phyB activity ${ }^{47}$. We used 35S::PHYB-GFP lines to monitor day length and temperature contribution to phyB nuclear dynamics. Plants were grown in short days and $22^{\circ} \mathrm{C}$ or $28^{\circ} \mathrm{C}$ for 4 days and, on the fifth day, confocal images were obtained from the middle hypocotyl every two hours, for a $24 \mathrm{~h}$ interval (Fig. 2G). The number and size of nuclear bodies were quantified in these images (Supplemental Fig. 3, Methods), and active phyB levels (Fig. 2C) extrapolated by multiplying total nuclear fluorescence (Supp. Fig. 3A) by nuclear bodies mean size (Supp. Fig. 3B). phyB-GFP fluorescence rapidly localized into discrete nuclear foci after $2 \mathrm{~h}$ exposure to light (Fig. $2 G)$, with size of these foci gradually increasing throughout the day and their number reduced. Larger photobodies $(>0.45-0.28 \mu \mathrm{m})$ declined after day-to-night transition (Supp Fig. 3D-F), and their disassembly into smaller foci was accelerated towards the second half of the night (Supp. Fig. $3 \mathrm{G}-\mathrm{I}$ ). At $28^{\circ} \mathrm{C}$, the number of $0.45-0.28 \mu \mathrm{m}$ photobodies per nuclei was severely reduced as compared to $22^{\circ} \mathrm{C}$ (Fig. 2G, Supp. Fig. 3D-F). This reduction, however, was not accompanied by a greater abundance of smaller foci during the day, but only during early night (Supp. Fig. 3G-I). Quantification of total nuclear fluorescence showed that phyB abundance is substantially reduced at $28^{\circ} \mathrm{C}$ (Fig. $3 A)$, although diurnal changes in phyB-GFP fluorescence were not affected by temperature. Indeed, phyB-GFP levels remained roughly constant at $22^{\circ} \mathrm{C}$ in the day, and slowly declined at night, with an equivalent pattern observed at $28^{\circ} \mathrm{C}$, except for a faster decline at night (Supp. Fig. 3A).

Temperature reduces phyB stability at a post-transcriptional level, since these lines expressed the phyB-GFP protein under control of the constitutive $35 S$ promoter. Related temperature-dependent changes in phyB stability were reported recently ${ }^{45}$, although temperature treatments and results differed from ours. Active phyB levels steadily increased during the day in our growth conditions and reached maximal levels shortly 
before dusk, to exponentially decay at night (Fig. 2C). phyB was also found to be less abundant at $28^{\circ} \mathrm{C}$, and large photobodies undergo fastest transition into smaller foci during early night (Fig. 2C). The model captures these dynamics, as it predicts that bioactive phyB is reduced at warm temperatures, and more rapidly inactivated in the dark (Fig. 2F).

\section{COP1 regulates thermomorphogenesis in the light}

COP 1 function is essential for thermal elongation ${ }^{31}$, as reflected by the severely impaired thermoresponse of cop1-4 genotypes (Fig. 1A). COP1 was shown to accumulate in the nucleus at $28^{\circ} \mathrm{C}$, but higher activity in warm conditions to be uncoupled from light and timing information ${ }^{25}$. Contrarily to these initial observations, our mathematical model predicts that COP1 activity is close to saturation and unresponsive to temperature at night, whereas its function is reduced during the day, being then critical for temperatureresponsive HY5 turnover (Fig. 3A). We therefore simulated hypocotyl growth for a range of values of COP1 activity, and assigned the weak cop1-4 alleles as a 100-fold reduction in activity, and COP1-OE lines as a 100-fold increase (Fig. 3B). Running the model with these arbitrary parameters captured thermal behavior of these genotypes, as it showed that reduced activity of cop1-4 mutants leads to defective elongation, and an alikeimpaired thermoresponse in darkness as in CWL. Ectopic COP1 expression, by contrast, is predicted to be of no effect in darkness, but lead to taller hypocotyls and an enhanced thermoresponse in CWL, as illustrated by a remarkable increase in hypocotyl length at $28^{\circ} \mathrm{C}$ (Fig. 3B).

Growth of COP1-OE and hy5 lines, as compared to Col-0, follows this behavior (Fig. 1A; Supp. Fig. 1A and 1B), consistent with COP1 acting at temperature signaling in the light. We therefore analyzed the light-dependent thermoresponse of independent COP1 overexpressors by using 35S::YFP-COP1 ${ }^{48}$, UBQ::RFP-COP1 and COP1-OE 49 lines. Although these plants accumulate increasing levels of COP1 (Fig. 3C and 3D), their hypocotyl lengths were identical to the wild type in the dark, and failed to elongate further 
at $28^{\circ} \mathrm{C}$ (Fig. $3 \mathrm{C}$ and $3 \mathrm{D}$ ). However, in $\mathrm{CWL}$ they displayed a thermal elongation response that was directly proportional to COP1 abundance (Fig. 3B). An identical behavior was observed in continuous red (CRL) and blue light (CBL) (Supp. Fig. 4A), in support of this response being independent of phyB Pfr thermal inactivation. Furthermore, COP1 abundance was reduced in etiolated seedlings (Fig. 3E), suggesting that COP1 auto-degradation is suppressed in the light.

Thermal Pfr inactivation is presumed to enable COP1-SPA1 re-assembly, and thereby favor COP1 activity in CRL. Modeling thermal behavior of phyB-9 COP1-OE and PHYBox COP1-OE lines actually predicted an identical growth phenotype for $P H Y B o x$ COP1-OE (Fig. 3F) and PHYBox plants (Fig. 1A), while phyB-9 COP1-OE lines to exhibit a constitutively tall phenotype, irrespective of temperature and day length conditions (Fig. 3G). Experimental validation of these phenotypes showed that $P H Y B o x$ COP1-OE lines display, as predicted, short hypocotyls in the light and a hypersensitive response to day length thermal inhibition (Fig. 3G). However, they thermoelongate in short days and $4 \mathrm{~h}$ light cycles, indicating that temperature inactivates only a fraction of the Pfr pool and remaining Pfr exceeds COP1 and PIFs titration levels. On the other side, phyB-9 COP1$O E$ lines showed as predicted a tall phenotype in CWL, but retained a residual response to day length and temperature (Fig. $3 F$ and $3 G$ ). These plants were in CWL taller than the rest of genotypes, but hypocotyl growth was partially suppressed as day length increased, and enhanced at $28^{\circ} \mathrm{C}$. This behavior can be explained by the action of other photoreceptors not considered in the model. The blue light receptors cryptochromes (CRY1 and CRY2), for instance, disrupt COP1-SPA interaction, in addition to displace COP1 binding to its degradation targets via a conserved VP motif ${ }^{50-52}$. CRY1 likewise interacts in a blue light-dependent manner with PIF4, suppressing PIF4-mediated hypocotyl elongation at elevated temperatures ${ }^{53}$. In line with this notion, deviation of phyB-9 COP1-OE growth with respect to the model is accentuated in CBL, whereas significantly reduced in CRL (Supp. Fig. 4B). Opposing effects of red and blue light, thus 
support a phyB-independent role of COP1 in temperature signaling, this thermal role being additively enhanced by impaired phyB activity.

\section{Architecture of the thermomorphogenic network}

Although the COP1 protein accumulates in the nucleus at elevated temperatures ${ }^{25}$, biological relevance of this process remained an open question. Our modeling studies unveil a specific thermal signaling function of COP1 in the light, which most likely was masked in former studies. Fitting phyB thermal inactivation parameters ${ }^{4,5}$ to the gathered growth data enabled us to derive a minimal thermal growth model where only the essential interactions were included. This model shows that the thermal responsive network comprises a triple feed-forward coherent motif, whereby ELF3 and phyB, on one hand, and COP1, on the other, exert opposing roles on thermomorphogenic growth (Fig. 1B).

Finally, we used this minimal model to explore the parameter space of these coherent motifs, to delimit their relative action and effects of day length duration on their thermosensory role. Heatmaps showing the contribution of COP1, ELF3, and phyB (horizontal axis) to thermal elongation (growth at $28^{\circ} \mathrm{C}$ compared to $22^{\circ} \mathrm{C}$ ), and how these are predicted to vary with day length (vertical axis), are shown in Fig. 4. Also, linked effects of these motifs were inferred by generating similar heatmaps for the mutant backgrounds (as indicated in the panels). Red plot areas indicate conditions in which response to temperature is maximal, considering the combined effect of day length and activities of these regulators. For abscissa values $=1$, moving vertically on the ordinate axes shows that thermal elongation (color code on the right axis) is greater in short days (lower values in left axis), but significantly reduced in long days and CWL (upwards in left axis), as for our experimental data in Fig. 1A. Moving horizontally along a constant day length shows impact of changes in the different regulators on thermal elongation: i.e. for $\mathrm{a} 12 \mathrm{~h}$ day length, smaller abscissa values show that reduced COP1 levels abolish 
thermoelongation, while thermal growth is strongly enhanced in response to increased COP1 activity (higher abscissa values), in line with the cop1-4 and COP1-OE phenotypes in Fig. 1A. An opposite behavior is observed for phyB activity, highlighting an opposing role of phyB and COP1 in driving thermal elongation. Thermal effects of high COP1 activity are lost in the absence of phyB, consistent with the phenotype of phyB-9 COP1-OE lines (Fig. 3F-G). Defective COP1 function moreover impairs thermoelongation under any condition (Fig. 4, middle row, central and right panels), highlighting the key contribution of this regulator to thermomorphogenesis.

On the other side, ELF3 is predicted to have a main contribution to thermal growth in short days (Fig. 4, upper row, central panel), consistent with its role in driving PIF4 expression during nighttime. Loss of ELF3 function, weakens thermal effects of elevated COP1 or reduced phyB levels and narrows them down to longer day lengths. Moreover, phyB is found to override ELF3 activity, as reflected by the enhanced thermal growth, over a wider range of day lengths, caused by elevated ELF3, in the phyB-9 background (Fig. 4, lower central panel). Surprisingly, a small elevation in ELF3 is predicted to favor thermal elongation, whereas greater ELF3 levels reverse this positive effect (Fig. 4). LUX was actually shown to bind with high affinity the LBS recognition motif, whereas its activity would be abolished on dimerization with ELF3 and restored on additional interaction with ELF4 ${ }^{8}$. It is thus conceivable that ELF3 counteracts LUX inhibition of PIF4 and its own expression, until LUX elevation and higher EC levels reverse this positive effect. Even though this dual function remains to be substantiated, it is noteworthy that it is predicted to be potentiated by loss of phyB activity, hence underscoring a role of phyB signaling in antagonizing ELF3. Notably, COP1 thermal activity also appears in these simulations to be affected by the lack of ELF3, suggesting that ELF3 and COP1 function is somehow linked (Fig. 4, lower left panel). Thus, it will be interesting in future studies to validate these novel interactions and investigate their underlying molecular mechanisms. 


\section{DISCUSSION}

Thermal responsiveness is pivotal in nature to reduce the impact of potentially damaging temperatures. Warm-induced hypocotyl elongation drives the apical meristem above the warm soil, while upwards foliar orientation reduces the incidence of direct sun light and favors aeration to cool the leaves. Magnitude of this response is however reduced on exposure to full sunlight and longer day lengths of summer, which overlap with warmer temperatures. Understanding the molecular processes driving this inhibition is thus pivotal to optimize resilience to climate change of long day-requiring crops. In this work, we show that COP1 acts as a major thermal regulator in the light. COP1 was shown to accumulate in the nucleus at elevated temperatures ${ }^{25}$, our findings pointing to warm temperatures interfere with nuclear exclusion of the protein in the light. The photoreceptors phyB, phyA and cryptochromes bind SPAs and disrupt COP1-SPAs complex formation ${ }^{19,54}$. SPAs were reported to act as essential COP1 cofactors, being proposed to retain COP1 in the nucleus in the dark. This dual role has been however questioned by the observation that spaQn mutants normally accumulate COP1 in the nucleus in darkness, but fail at cytosolic COP1 relocation on exposure to light 55 . Thereby, when unbound to COP1, SPAs may take part of the nuclear COP1 exclusion machinery, having a central role in COP1 thermal regulation. COP1/SPA activity suppresses light signaling by targeting degradation of several light response factors antagonizing PIFs, including HY5 and HFR1. The COP1/SPA complex targets also ELF3 26,28 for degradation, and is required for enhanced PIF4 expression and PIF4 protein stabilization in warm conditions ${ }^{25,56}$. Therefore, it is conceivable that nuclear COP1 accumulation mediates enhanced thermal elongation through a combination of these processes.

As phyB and SPA1 interaction suppresses COP1 activity, it cannot be excluded that light-dependent COP1 thermal function is a primarily consequence of phyB Pfr thermal 
inactivation. Our results, however, do not support this mechanistic model. Loss of phyB or ELF3 function in the double phyB-9 cop1-4 and elf3-8 cop1-4 mutants is unable to rescue thermal elongation defects of cop1-4, revealing that COP1 activity is crucial for the enhanced thermal responsiveness of the phyB-9 and elf3-8 backgrounds (Fig. 1A). Positive effects of COP1 in CWL are additive to the phyB-9 mutation, suggesting that thermal COP1 function is to an important extent independent of phyB activity. Yet COP1$O E$ phyB-9 lines were not as elongated in CWL as predicted by the model (Fig. 3G), this divergence was notably reduced in $C R L$, while it was aggravated in $C B L$ (Supp. Fig. 4B). This supports that this phenotype is caused by direct COP1 inhibition by the blue light receptor cryptochromes 57,58, calling for future improvement of the model, by incorporating these photoreceptors

Thermoelongation heatmaps (Fig. 4) predict that phyB and COP1 activities are more relevant as day length increases, while role of ELF3 is more prominent in short days. Both reduced phyB and higher COP1 levels lead to significantly enhanced thermal elongation in long days and CWL, while defective COP1 function abolishes thermal responsiveness irrespective of phyB and ELF3 levels. Therefore, the model fully captures the epistatic effects of cop1-4 on loss of phyB or ELF3 function, supporting a pivotal role of COP1/HY5 in modulating PIF4 activity downstream of these regulators. Heatmap simulations also show that impaired ELF3 activity reduces phyB and COP1 thermal effects, consistent with defective EC function leading to a de-repressed response at $22^{\circ} \mathrm{C}$. Model predictions thus substantiate the phenotypes of double overexpressor/ mutant lines, demonstrating that the modelled interactions are sufficient to explain Arabidopsis thermal behavior.

Temporal dynamics of ELF3 and PIF4 abundance, as measured using pELF3::ELF3LUC and pPIF4::PIF4-LUC lines, showed that accumulation of these proteins, in particular PIF4, is strongly affected by day length. PIF4 expression rises in short days during late night, while in long days it is elevated only during daytime (Fig. 2). Likewise, 
warm temperatures delay in long days the peak of PIF4 transcription during late evening, leading to a marginal increase of PIF4 protein levels at dusk (Supp. Fig. $2 \mathrm{l}$ and $2 \mathrm{H}$ ). COP1 was recently reported to destabilize the DELLA repressors at warmer temperatures ${ }^{59}$ and in this regard, it is possible that this control takes on a central role in longer day lengths. While diurnal ELF3 oscillation was coarsely introduced when fitting the model, PIF4 activity was remarkably left unconstrained. However, in its current configuration the model correctly predicts that PIF4 activity is enhanced at elevated temperatures, while this effect is greater in short days as compared to longer day lengths. Thus, future up-grading of the model by using dynamics of these regulators as measured in the LUC lines, will certainly help to its fine-tuning and improve its already strong predictive power.

Most crop species show a narrow genetic variability in thermomorphogenic-related traits ${ }^{60}$, which threats their productivity in a global warming scenario. Natural variation in the phyB and ELF3 loci was found to be associated with increased thermal sensitivity in Arabidopsis and several crops, in a consistent manner with their adaptation to local climate variables $6,9,61,62$. Lower activity of these alleles results in an elongated phenotype that is also associated with earlier flowering, and thereby leads to a reduction in yield. We here show that COP1 activity is essential for increased thermal responsiveness of phyB-9 and elf3-8 mutants, and that COP1 over-expression leads to a significantly enhanced thermomorphogenic growth in the light, but a wild-type response in darkness. COP1-OE lines, on the other hand, do not exhibit the same accelerated flowering as phyB-9 and elf3-8 mutants. Hence, allelic variants in the COP1 gene that lead to an overall increase in protein levels or activity emerge from this work as excellent candidates for optimizing crops resilience to climate change. 


\section{MATERIALS AND METHODS}

\section{Plant materials}

Arabidopsis thaliana Col-0 plants were used as wild type (WT) in this study. The mutants and transgenic lines used in the different experiments were: elf3-8 ${ }^{63}$, ELF30x ${ }^{26}$, pif4$101^{64}$, pifq ${ }^{65}$, phyB-9 ${ }^{66}$, COP10x ${ }^{26}$ COP1-OE ${ }^{49}$, cop1-4 ${ }^{67}$, hy5-215 ${ }^{68}$, elf3-8 cop1-4 ${ }^{26}$, ELF3ox cop1-4 26, PIF4-HA 69, pELF3::ELF3-myc 7, pPIF4::PIF4-HA 42, cop1-4/ 35S:YFP-COP1 ${ }^{48}$, 35S:PHYB-GFP ${ }^{70}$.

\section{Growth conditions}

Seeds were surface-sterilized for 15 minutes in 70\% (v/v) ethanol and 0,1\% (v/v) Tween 20 , followed by two washes of 2 minutes in $96 \%(v / v)$ ethanol. Seeds were air dried and sown on half strength MS-agar plates with $1 \%$ sucrose and stratified for 3 days at $4^{\circ} \mathrm{C}$ in the dark. Germination was synchronized by illuminating the plates with white light for 4 hours and transferring them back to darkness for 20 additional hours. The seedlings were then grown in specific temperature and day length conditions with white light (50 $\left.\mu \mathrm{molm}{ }^{-2} \mathrm{~s}^{-1}\right)$, red light $\left(35 \mu \mathrm{molm}^{-2} \mathrm{~s}^{-1}\right)$ or blue light $\left(35 \mu \mathrm{molm} \mathrm{m}^{-2} \mathrm{~s}^{-1}\right)$.

\section{Plasmid constructs and generation of Arabidopsis transgenic lines}

To generate the pELF3::LUC expression cassette, a fragment containing $2.21 \mathrm{~kb}$ upstream regulatory region of ELF3 was amplified from Col-0 genomic DNA using primers ELF3Prom-Fw (5'-CACCCTTATAAATAAAATTCC-3') ELF3Prom-Rv (5'CACTCACAATTCACAACCTTTTTC-3') and cloned into the Gateway System (pENTR ${ }^{\text {TM }}$ Directional TOPO ${ }^{\circledR}$ Cloning kit, Invitrogen) to obtain the pELF3-TOPO plasmid. To generate $p E L F 3:: E L F 3-L U C$ construct, the ELF3 coding region was amplified without the stop codon using the ELF3 cloning-Fw/ ELF3 cloning-Rv primers and cloned into pENTR ${ }^{T M} / D-T O P O$ vector. The PCR fragment of $p E L F 3$ sequence was amplified from pELF3-TOPO with ELF3 Prom Notl-Fw/ ELF3 Prom Notl-Rv and then inserted into Notl of ELF3-TOPO, to produce the pELF3::ELF3-TOPO plasmid. Finally, pELF3-TOPO and 
pELF3::ELF3-TOPO constructs were mobilized by LR clonase II recombination (Invitrogen) into the pLUC-Trap3 vector ${ }^{71}$.

To generate the pPIF4::LUC reporter gene, we amplified a fragment $2.47 \mathrm{~kb}$ upstream of the PIF4 ATG with primers PIF4Prom-Fw (5'-CACCCAGTACGCATCCAATCTTCTC3') and PIF4Prom-Rv (5'-CGGGATCCGGGTACAGACAGAAAGTGAC-3') and followed

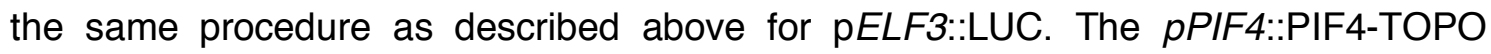
construct ${ }^{42}$ was transferred to the pLUC-Trap3 destination vector.

COP1 CDS was amplified using primers COP1cloning-Fw (5'-CACCATGGAAGAGA TTTCGACGGATC-3') and COP1cloning-Rv (5'-GAAGATCTTCACGCAGCGAGTACC

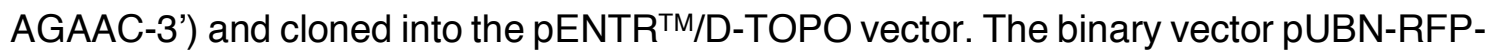
Dest ${ }^{72}$ was used for N-terminal fusion of the mRFP tag.

To generate Arabidopsis transgenic lines, the Agrobacterium-mediated floral dip method was used to transform pELF3-LUC, pELF3::ELF3-LUC, pPIF4-LUC and pPIF4::PIF4LUC plasmids into Col-0, whereas pUBN-RFP-COP1 was transformed into cop1-4 mutant background. Homozygous T3 plants were used in this study.

The double homozygous phyB-9 COP1-OE and PHYBOx COP1-OE were generated by crossing the transgenic COP1-OE line with phyB-9 and $P H Y B O x$, respectively. The phyB9 mutation was genotyped using primers phyB-9-fw (5'-GCAA TGCCACACCTGTTCTTGTGG'3') and phyB-9-rv (5'-CTTCACTAGGAGCAACACCCA ACG-3'). phyB and COP1 over-expression constructs were PCR-genotyped with primers 35S-fw (5'-CCACTGACGTAAGGGATGAC-3') and phyB-9-rv and COP1-PE-fw (5'CTTCCCTCCGTACTACACTCTTATC-3'), respectively.

\section{Hypocotyl measurements}

Seeds were sown on half strength MS-agar plates with $1 \%$ sucrose and stratified 4 days at $4^{\circ} \mathrm{C}$ in darkness. After stratification, the germination was induced by placing the plates for $4 \mathrm{~h}$ under $50 \mu \mathrm{mol} . \mathrm{m}^{-2} . \mathrm{s}^{-1}$ white light and $20 \mathrm{~h}$ darkness at $22^{\circ} \mathrm{C}$. After 4 days at the 
indicated photoperiod and temperature regimes, 20-30 seedlings were photographed and hypocotyl length was determined using ImageJ software.

\section{Luciferase activity assays}

Luciferase activity assays were carried out using the LB 960 Microplate Luminometer (Berthold Technologies, UK). Transgenic Arabidopsis seedlings were plated on $0.5 \mathrm{x}$ MS-agar medium and, after 2-3 days of germination, they were transferred to 96 well microplates (Costar, USA) filled with $175 \mu \mathrm{L} 0.5 \times$ MS-agar medium $1 \%$ sucrose and $35 \mu \mathrm{L}$ (50 $\mu \mathrm{g} / \mathrm{mL}$ ) of Beetle Luciferin (Promega) dissolved in DMSO. Levels of luciferase activity were registered every hour and represented as the average of counts per two seconds in each well, with at least 24 plants per line. The luminometer was housed in a growth chamber to maintain the plants under controlled growth conditions.

\section{Confocal microscopy}

Confocal fluorescence images of phyB-GFP were taken from the epidermis and the first sub-epidermal layers of the upper third portion of the hypocotyl. We used a LSM5 Pascal laser scanning microscope (Zeiss) with a water-immersion objective lens (C-Apochromat 40x/1,2; Zeiss). Probes were excited with an Argon laser $(\lambda=488 \mathrm{~nm})$ and fluorescence was detected using a BP 505-530 filter. Images were taken from individual nuclei and image analysis was performed as in Legris et al., 2016. Each data point consists of the average of 6 replicates coming from two independent experiments showing the same trend. Replicates consist of the average of 3 plants coming from the same plate, and 3 nuclei per plant were analyzed.

\section{Western blot analysis}

Total protein extracts from equal number of Arabidopsis seedlings were prepared by homogenizing plant material in extraction buffer containing $125 \mathrm{mM}$ Tris $\mathrm{HCl} \mathrm{pH} \mathrm{7.4,} \%$ SDS, $10 \%$ glycerol, $6 \mathrm{M}$ urea and $1 \% \beta$-mercaptoethanol. The extracts were heated at $95^{\circ} \mathrm{C}$ for $3 \mathrm{~min}$ and then centrifuged at $4^{\circ} \mathrm{C}$ for $15 \mathrm{~min}$ to recover the supernatant. Protein samples were boiled for 5 min in TMx2 loading buffer, and $40 \mu \mathrm{L}$ of the protein extracts 
run on $8 \%$ SDS-PAGE gels. Homogeneous protein transfer to nitrocellulose membranes (Whatman) was confirmed by Ponceau red staining. For PIF4 and ELF3 detection, blots were respectively immunodetected with an anti-HA Peroxidase (Roche) or anti-myc antibody (Abmart), followed by incubation with anti-mouse HRP-conjugated antibody. Anti-RPT5 (Enzo) was used for equal loading control. For COP1 immunodetection it was used an anti-COP1 antibody kindly supplied by Dr. Hoecker 73 followed by incubation with a HRP-conjugated anti-rabbit secondary antibody. Chemiluminescence was detected with the Supersignal West Pico and Femto substrates (Pierce). 


\section{FIGURE LEGENDS}

Figure 1. Phenotype of loss-of function mutants and overexpressors under different temperatures and day lengths.

(A) Growth of twelve Arabidopsis genotypes at either $22^{\circ} \mathrm{C}$ or $28^{\circ} \mathrm{C}$ under six white light day length conditions. Measured hypocotyl lengths (circles) are compared with values predicted by the growth model (solid lines). Bars indicate standard deviation $(n=30)$. The model accurately captures the main trends of data. (B) Schematic representation of the network tested. Dashed and solid lines indicate transcriptional and post transcriptional regulations respectively.

\section{Figure 2. Predicted and observed dynamics of ELF3, PIF4 and phyB.}

Bioluminescence assays of Col-0 lines expressing constructs $\mathrm{pELF3::ELF3-LUC} \mathrm{(A)} \mathrm{and}$ pPIF4::PIF4-LUC (B). Seedlings were grown in short day cycles at the indicated temperatures. Values represent mean \pm SEM of absolute bioluminescence of at least 24 seedlings. Bioluminescence of seedlings was measured every hour. (D) ELF3 and (E) PIF4 activity predicted by the model. (C and $\mathbf{G}$ ) Temperature effects on phyB nuclear bodies in short day conditions. phyB-GFP transgenic seedlings were grown in short day cycles at either $22^{\circ} \mathrm{C}$ or $28^{\circ} \mathrm{C}$ for 5 days. Mean size of phyB nuclear bodies and total nuclear fluorescence measured with the Matlab software from confocal images (C) and diagram of sampling time points and growth conditions (G). Arbitrary units (a.u.) of phyB activity were calculated by multiplying the nuclear bodies mean size by nuclear fluorescence at each sampling time point. Ten seedlings were measured in each assay and two biological replicates were analyzed. Warmer temperature results in fewer photobodies and less nuclear intensity. All seedlings were grown under $50 \mu \mathrm{mol} . \mathrm{m}^{-2} . \mathrm{s}^{-1}$ white light in short day cycles at $22^{\circ} \mathrm{C}$ or $28^{\circ} \mathrm{C}$. (F) phyB activity predicted by the model. 
Rectangles indicate light conditions: white, lights on, and grey, lights off. ZT indicates the zeitgeber time.

\section{Figure 3. Effect of changes in COP1 activity on thermal growth}

(A) Levels of COP1 activity predicted by the mathematical model in short day conditions.

(B) Hypocotyl length as affected by the interaction of environmental conditions and COP1 levels. Observed hypocotyl lengths of Col-0, cop1-4 and COP1-OE (symbols) versus the growth values estimated by the model (solid lines) in dark (left panel) and CWL (right panel). (C) Hypocotyl length phenotypes of different COP1 overexpressors. Bars indicate standard deviation ( $n=30$ ). (D) Pictures of five-days-old seedlings grown in CWL (upper panel) and continuous darkness (lower panel) at $22^{\circ} \mathrm{C}$ or $28^{\circ} \mathrm{C}$. Bar $=10$ $\mathrm{mm}$. (E) Protein blot showing that increased accumulation of the COP1 protein correlate with enhanced thermal elongation in CWL, but this response is saturated in darkness. COP1 was detected using an anti-COP1 antibody. RPT5 was used for loading control and detected using an anti-RPT5 antibody. Total protein extracts were used for immunoblot analysis. (F) Phenotypes of phyB-9 COP1-OE and PHYBox COP1-OE seedlings grown for five days either in darkness or $\mathrm{CWL}$ at $22^{\circ} \mathrm{C}$ or $28^{\circ} \mathrm{C}$. (G) Observed hypocotyl growth (circles) of phyB-9 COP1-OE and PHYBox COP1-OE) lines grown at different day lengths and $22^{\circ} \mathrm{C}$ or $28^{\circ} \mathrm{C}$. Solid lines are predictions from the model.

Figure 4. phyB, COP1 and ELF3 contribution to day length-dependent hypocotyl thermoelongation as predicted by the model.

Heatmap plots representing hypocotyl thermoelongation (calculated as the difference of hypocotyl length at $28^{\circ} \mathrm{C}$ and $22^{\circ} \mathrm{C}$ ) relative to day length ( $\mathrm{Y}$ axis) and activity of the COP1, phyB and ELF3 proteins ( $\mathrm{X}$ axis, as indicated). $\mathrm{A}$ value $=1.0$ corresponds to wild type level, while greater and lesser values are equivalent to overexpression and loss-offunction, respectively. Backgrounds are indicated in the panels. 


\section{Supplementary figure 1. Model predictions for hypocotyl thermoelongation of backgrounds or day length conditions different to those used to fit the model.}

(A) Growth of three Arabidopsis genotypes at either $22^{\circ} \mathrm{C}$ or $28^{\circ} \mathrm{C}$ under five white light day length conditions. (B) Growth of six Arabidopsis genotypes in a $4 \mathrm{~h}$ light regime and either $22^{\circ} \mathrm{C}$ or $28^{\circ} \mathrm{C}$. Measured hypocotyl length (circles) were compared with values predicted by the thermoresponse model (solid lines). Lines are model predictions and not fits to the data. Bars indicate standard deviation $(n=30)$.

\section{Supplementary figure 2. Effect of warm temperature on ELF3 and PIF4 expression and protein abundance under short and long days.}

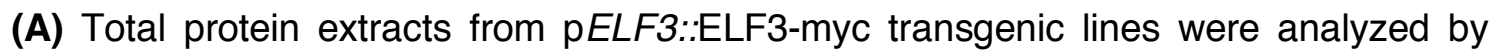
Western blot using an anti-myc antibody. RPT5 was used for loading control and was detected using an anti-RPT5 antibody. Seedlings were grown for 7 days at $22^{\circ} \mathrm{C}$ or $28^{\circ} \mathrm{C}$ under short day conditions. Samples were harvested at the indicated ZT. Bioluminescence assays of Col-0 lines expressing ELF3-LUC (B) and PIF4-LUC (G) in short days. Bioluminescence recorded from transgenic seedlings expressing the pELF3::ELF3-LUC (C), pELF3::LUC (D), pPIF4::PIF4-LUC (H) and pPIF4::LUC (I) constructs in long days at $22^{\circ} \mathrm{C} / 28^{\circ} \mathrm{C}$. Values represent mean $\pm \mathrm{SE}$ of absolute bioluminescence of at least 24 seedlings. (E) ELF3 and (J) PIF4 activity predicted by the model. (F) Western blot of pPIF4::PIF4-HA transgenic lines. The PIF4-HA protein was detected using an anti-HA antibody. Ponceau staining was used as loading control. Rectangles indicate light conditions: white, lights on, and grey, lights off. ZT indicates the zeitgeber time.

Supplementary figure 3. phyB nuclear bodies formation is affected by temperature in short day conditions.

35S::PHYB-GFP transgenic seedlings were grown under $50 \mu \mathrm{mol} . \mathrm{m}^{-2} . \mathrm{s}^{-1}$ white light in short day cycles at $22^{\circ} \mathrm{C}$ or $28^{\circ} \mathrm{C}$ for 7 days. (A) Total nuclear fluorescence expressed in 
arbitrary units (a.u.). (B) Nuclear photobodies mean size $\left(\mu \mathrm{m}^{2}\right)$. (C) Number of total bodies per nucleus. (D-I) Number of phyB nuclear bodies, sorted by size categories, in $\mu \mathrm{m}$, measured with Matlab Software. The rectangles indicate the light conditions: white, lights on and grey, lights off. ZT, zeitgeber time.

\section{Supplementary figure 4. Thermal response of COP1 lines in red and blue light.}

(A) Hypocotyl length phenotypes of the different COP1 overexpressor lines in continuous red (CRL) and continuous blue (CBL) light. (B) Phenotypes of phyB-9 COP1OE and PHYBox COP1-OE seedlings grown for five days either in darkness, CWL, CRL and $\mathrm{CBL}$, at $22^{\circ} \mathrm{C}$ or $28^{\circ} \mathrm{C}$. Bars indicate standard deviation $(n=30)$.

\section{References}

1. Burgie, E. S. \& Vierstra, R. D. Phytochromes: an atomic perspective on photoactivation and signaling. Plant Cell 26, 4568-4583 (2014).

2. Casal, J. J. Photoreceptor Signaling Networks in Plant Responses to Shade. Annu. Rev. Plant Biol. 64, 403-427 (2013).

3. Gommers, C. M. M., Visser, E. J. W., Onge, K. R. S., Voesenek, L. A. C. J. \& Pierik, R. Shade tolerance: when growing tall is not an option. Trends in Plant Science 18, 65-71 (2013).

4. Jung, J.-H. et al. Phytochromes function as thermosensors in Arabidopsis. Science 354, 886-889 (2016).

5. Legris, M. et al. Phytochrome B integrates light and temperature signals in Arabidopsis. Science 354, 897-900 (2016).

6. Jung, J.-H. et al. A prion-like domain in ELF3 functions as a thermosensor in Arabidopsis. Nature 585, 256-260 (2020).

7. Ezer, D. et al. The evening complex coordinates environmental and endogenous signals in Arabidopsis. Nat Plants 3, 17087 (2017).

8. Silva, C. S. et al. Molecular mechanisms of Evening Complex activity in Arabidopsis. Proc. Natl. Acad. Sci. U.S.A. 117, 6901-6909 (2020).

9. Box, M. S. et al. ELF3 controls thermoresponsive growth in Arabidopsis. Curr. Biol. 25, 194-199 (2015).

10. Koini, M. A. et al. High temperature-mediated adaptations in plant architecture require the bHLH transcription factor PIF4. Curr. Biol. 19, 408-413 (2009).

11. Fiorucci, A.-S. et al. PHYTOCHROME INTERACTING FACTOR 7 is important for early responses to elevated temperature in Arabidopsis seedlings. New Phytol. 226, 50-58 (2020).

12. Pham, V. N., Xu, X. \& Huq, E. Molecular bases for the constitutive photomorphogenic phenotypes in Arabidopsis. Development 145, (2018).

13. Park, E., Kim, Y. \& Choi, G. Phytochrome B Requires PIF Degradation and Sequestration to Induce Light Responses across a Wide Range of Light Conditions. 
The Plant Cell 30, 1277-1292 (2018).

14. Helfer, A. et al. LUX ARRHYTHMO encodes a nighttime repressor of circadian gene expression in the Arabidopsis core clock. Curr. Biol. 21, 126-133 (2011).

15. Nusinow, D. A. et al. The ELF4-ELF3-LUX complex links the circadian clock to diurnal control of hypocotyl growth. Nature 475, 398-402 (2011).

16. Osterlund, M. T., Wei, N. \& Deng, X. W. The roles of photoreceptor systems and the COP1-targeted destabilization of HY5 in light control of Arabidopsis seedling development. Plant Physiol. 124, 1520-1524 (2000).

17. Duek, P. D., Elmer, M. V., van Oosten, V. R. \& Fankhauser, C. The degradation of HFR1, a putative bHLH class transcription factor involved in light signaling, is regulated by phosphorylation and requires COP1. Curr. Biol. 14, 2296-2301 (2004).

18. Yang, J. et al. Repression of light signaling by Arabidopsis SPA1 involves posttranslational regulation of HFR1 protein accumulation. Plant J. 43, 131-141 (2005).

19. Lu, X.-D. et al. Red-light-dependent interaction of phyB with SPA1 promotes COP1-SPA1 dissociation and photomorphogenic development in Arabidopsis. Mol Plant 8, 467-478 (2015).

20. Subramanian, C. et al. The Arabidopsis repressor of light signaling, COP1, is regulated by nuclear exclusion: mutational analysis by bioluminescence resonance energy transfer. Proc. Natl. Acad. Sci. U.S.A. 101, 6798-6802 (2004).

21. Hornitschek, P., Lorrain, S., Zoete, V., Michielin, O. \& Fankhauser, C. Inhibition of the shade avoidance response by formation of non-DNA binding bHLH heterodimers. EMBO J. 28, 3893-3902 (2009).

22. Shi, H. et al. HFR1 sequesters PIF1 to govern the transcriptional network underlying light-initiated seed germination in Arabidopsis. Plant Cell 25, 3770-3784 (2013).

23. Chen, D. et al. Antagonistic basic helix-loop-helix/bZIP transcription factors form transcriptional modules that integrate light and reactive oxygen species signaling in Arabidopsis. Plant Cell 25, 1657-1673 (2013).

24. Toledo-Ortiz, G. et al. The HY5-PIF regulatory module coordinates light and temperature control of photosynthetic gene transcription. PLoS Genet. 10, e1004416 (2014).

25. Park, Y.-J., Lee, H.-J., Ha, J.-H., Kim, J. Y. \& Park, C.-M. COP1 conveys warm temperature information to hypocotyl thermomorphogenesis. New Phytol. 215, 269280 (2017).

26. Yu, J.-W. et al. COP1 and ELF3 control circadian function and photoperiodic flowering by regulating GI stability. Mol. Cell 32, 617-630 (2008).

27. Jang, I.-C., Henriques, R., Seo, H. S., Nagatani, A. \& Chua, N.-H. Arabidopsis PHYTOCHROME INTERACTING FACTOR proteins promote phytochrome B polyubiquitination by COP1 E3 ligase in the nucleus. Plant Cell 22, 2370-2383 (2010). 28. Nieto, C., López-Salmerón, V., Davière, J.-M. \& Prat, S. ELF3-PIF4 interaction regulates plant growth independently of the Evening Complex. Curr. Biol. 25, 187-193 (2015).

29. Al-Sady, B., Ni, W., Kircher, S., Schäfer, E. \& Quail, P. H. Photoactivated phytochrome induces rapid PIF3 phosphorylation prior to proteasome-mediated degradation. Mol. Cell 23, 439-446 (2006).

30. Ni, W. et al. A mutually assured destruction mechanism attenuates light signaling in Arabidopsis. Science 344, 1160-1164 (2014).

31. Delker, C. et al. The DET1-COP1-HY5 pathway constitutes a multipurpose signaling module regulating plant photomorphogenesis and thermomorphogenesis. Cell Rep 9, 1983-1989 (2014).

32. Ling, J.-J., Li, J., Zhu, D. \& Deng, X. W. Noncanonical role of Arabidopsis COP1/SPA complex in repressing BIN2-mediated PIF3 phosphorylation and degradation in darkness. Proc. Natl. Acad. Sci. U.S.A. 114, 3539-3544 (2017). 
33. Pham, V. N., Kathare, P. K. \& Huq, E. Phytochromes and Phytochrome Interacting Factors. Plant Physiol. 176, 1025-1038 (2018).

34. Huang, $\mathrm{H}$. et al. Identification of Evening Complex Associated Proteins in Arabidopsis by Affinity Purification and Mass Spectrometry. Mol. Cell Proteomics 15, 201-217 (2016).

35. Covington, M. F. et al. ELF3 modulates resetting of the circadian clock in Arabidopsis. Plant Cell 13, 1305-1315 (2001).

36. Podolec, R. \& Ulm, R. Photoreceptor-mediated regulation of the COP1/SPA E3 ubiquitin ligase. Current Opinion in Plant Biology 45, 18-25 (2018).

37. De Caluwé, J. et al. A Compact Model for the Complex Plant Circadian Clock. Frontiers in Plant Science 7, 74 (2016).

38. Johansson, H. et al. Arabidopsis cell expansion is controlled by a photothermal switch. Nat Commun 5, 4848 (2014).

39. Hoecker, U. The activities of the E3 ubiquitin ligase COP1/SPA, a key repressor in light signaling. Curr. Opin. Plant Biol. 37, 63-69 (2017).

40. Mizuno, T. et al. Ambient temperature signal feeds into the circadian clock transcriptional circuitry through the EC night-time repressor in Arabidopsis thaliana.

Plant Cell Physiol. 55, 958-976 (2014).

41. Yamashino, T. et al. Verification at the protein level of the PIF4-mediated external coincidence model for the temperature-adaptive photoperiodic control of plant growth in Arabidopsis thaliana. Plant Signal Behav 8, e23390 (2013).

42. Bernardo-García, S. et al. BR-dependent phosphorylation modulates PIF4 transcriptional activity and shapes diurnal hypocotyl growth. Genes Dev. 28, 16811694 (2014).

43. Seaton, D. D. et al. Linked circadian outputs control elongation growth and flowering in response to photoperiod and temperature. Mol. Syst. Biol. 11, 776 (2015).

44. de Lucas, M. et al. A molecular framework for light and gibberellin control of cell elongation. Nature 451, 480-484 (2008).

45. Hahm, J., Kim, K., Qiu, Y. \& Chen, M. Increasing ambient temperature progressively disassembles Arabidopsis phytochrome B from individual photobodies with distinct thermostabilities. Nat Commun 11, 1660 (2020).

46. Van Buskirk, E. K., Reddy, A. K., Nagatani, A. \& Chen, M. Photobody Localization of Phytochrome B Is Tightly Correlated with Prolonged and LightDependent Inhibition of Hypocotyl Elongation in the Dark. Plant Physiol. 165, 595-607 (2014).

47. Van Buskirk, E. K., Decker, P. V. \& Chen, M. Photobodies in light signaling. Plant Physiol 158, 52-60 (2012).

48. Oravecz, A. et al. CONSTITUTIVELY PHOTOMORPHOGENIC1 is required for the UV-B response in Arabidopsis. Plant Cell 18, 1975-1990 (2006).

49. Lin, X.-L. et al. An Arabidopsis SUMO E3 Ligase, SIZ1, Negatively Regulates Photomorphogenesis by Promoting COP1 Activity. PLOS Genetics 12, e1006016 (2016).

50. Liu, B., Zuo, Z., Liu, H., Liu, X. \& Lin, C. Arabidopsis cryptochrome 1 interacts with SPA1 to suppress COP1 activity in response to blue light. Genes Dev 25, 10291034 (2011).

51. Zuo, Z., Liu, H., Liu, B., Liu, X. \& Lin, C. Blue light-dependent interaction of CRY2 with SPA1 regulates COP1 activity and floral initiation in Arabidopsis. Curr Biol 21, 841-847 (2011).

52. Ponnu, J. Molecular mechanisms suppressing COP1/SPA E3 ubiquitin ligase activity in blue light. Physiologia Plantarum 169, 418-429 (2020).

53. Ma, D. et al. Cryptochrome 1 interacts with PIF4 to regulate high temperaturemediated hypocotyl elongation in response to blue light. PNAS 113, 224-229 (2016).

54. Sheerin, D. J. et al. Light-activated phytochrome A and B interact with members 
of the SPA family to promote photomorphogenesis in Arabidopsis by reorganizing the COP1/SPA complex. Plant Cell 27, 189-201 (2015).

55. Balcerowicz, M., Kerner, K., Schenkel, C. \& Hoecker, U. SPA Proteins Affect the Subcellular Localization of COP1 in the COP1/SPA Ubiquitin Ligase Complex during Photomorphogenesis. Plant Physiol. 174, 1314-1321 (2017).

56. Gangappa, S. N. \& Kumar, S. V. DET1 and HY5 Control PIF4-Mediated Thermosensory Elongation Growth through Distinct Mechanisms. Cell Rep 18, 344351 (2017).

57. Lau, K., Podolec, R., Chappuis, R., Ulm, R. \& Hothorn, M. Plant photoreceptors and their signaling components compete for COP1 binding via VP peptide motifs. EMBO J. 38, e102140 (2019).

58. Ponnu, J., Riedel, T., Penner, E., Schrader, A. \& Hoecker, U. Cryptochrome 2 competes with COP1 substrates to repress COP1 ubiquitin ligase activity during Arabidopsis photomorphogenesis. Proc. Natl. Acad. Sci. U.S.A. (2019) doi:10.1073/pnas.1909181116.

59. Blanco-Touriñán, N. et al. COP1 destabilizes DELLA proteins in Arabidopsis. Proc. Natl. Acad. Sci. U.S.A. 117, 13792-13799 (2020).

60. Parent, B. \& Tardieu, F. Temperature responses of developmental processes have not been affected by breeding in different ecological areas for $17 \mathrm{crop}$ species. New Phytologist 194, 760-774 (2012).

61. Raschke, A. et al. Natural variants of ELF3 affect thermomorphogenesis by transcriptionally modulating PIF4-dependent auxin response genes. BMC Plant Biol. 15, 197 (2015).

62. $\mathrm{Bu}, \mathrm{T}$. et al. A critical role of the soybean evening complex in the control of photoperiod sensitivity and adaptation. Proc Natl Acad Sci U S A 118, e2010241118 (2021).

63. Hicks, K. A., Albertson, T. M. \& Wagner, D. R. EARLY FLOWERING3 Encodes a Novel Protein That Regulates Circadian Clock Function and Flowering in Arabidopsis. The Plant Cell 13, 1281-1292 (2001).

64. Huq, E. \& Quail, P. H. PIF4, a phytochrome-interacting bHLH factor, functions as a negative regulator of phytochrome $B$ signaling in Arabidopsis. EMBO J. 21, 24412450 (2002).

65. Leivar, P. et al. Multiple phytochrome-interacting bHLH transcription factors repress premature photomorphogenesis during early seedling development in darkness. Curr Biol 18, 1815-1823 (2008).

66. Reed, J. W., Nagpal, P., Poole, D. S., Furuya, M. \& Chory, J. Mutations in the gene for the red/far-red light receptor phytochrome B alter cell elongation and physiological responses throughout Arabidopsis development. Plant Cell 5, 147-157 (1993).

67. McNellis, T. W. et al. Genetic and molecular analysis of an allelic series of cop1 mutants suggests functional roles for the multiple protein domains. Plant Cell 6, 487500 (1994).

68. Oyama, T., Shimura, Y. \& Okada, K. The Arabidopsis HY5 gene encodes a bZIP protein that regulates stimulus-induced development of root and hypocotyl. Genes Dev 11, 2983-2995 (1997).

69. Lorrain, S., Allen, T., Duek, P. D., Whitelam, G. C. \& Fankhauser, C. Phytochrome-mediated inhibition of shade avoidance involves degradation of growthpromoting bHLH transcription factors. Plant J. 53, 312-323 (2008).

70. Yamaguchi, R., Nakamura, M., Mochizuki, N., Kay, S. A. \& Nagatani, A. Lightdependent Translocation of a Phytochrome B-GFP Fusion Protein to the Nucleus in Transgenic Arabidopsis. J Cell Biol 145, 437-445 (1999).

71. Calderon-Villalobos, L. I. A. et al. LucTrap Vectors Are Tools to Generate Luciferase Fusions for the Quantification of Transcript and Protein Abundance in Vivo. 
Plant Physiol 141, 3-14 (2006).

72. Grefen, C. et al. A ubiquitin-10 promoter-based vector set for fluorescent protein tagging facilitates temporal stability and native protein distribution in transient and stable expression studies. Plant J. 64, 355-365 (2010).

73. Balcerowicz, M. et al. Light exposure of Arabidopsis seedlings causes rapid destabilization as well as selective post-translational inactivation of the repressor of photomorphogenesis SPA2. Plant J. 65, 712-723 (2011). 
bioRxiv preprint doi: https://doi.org/10.1101/2021.11.25.470009; this version posted November 25, 2021. The copyright holder for this preprint (which was not certified by peer review) is the author/funder, who has granted bioRxiv a license to display the preprint in perpetuity. It is made available under aCC-BY-NC-ND 4.0 International license.

Figure 1. The phenotype of loss-of-function mutants and overexpressors under different temperatures and day lengths.
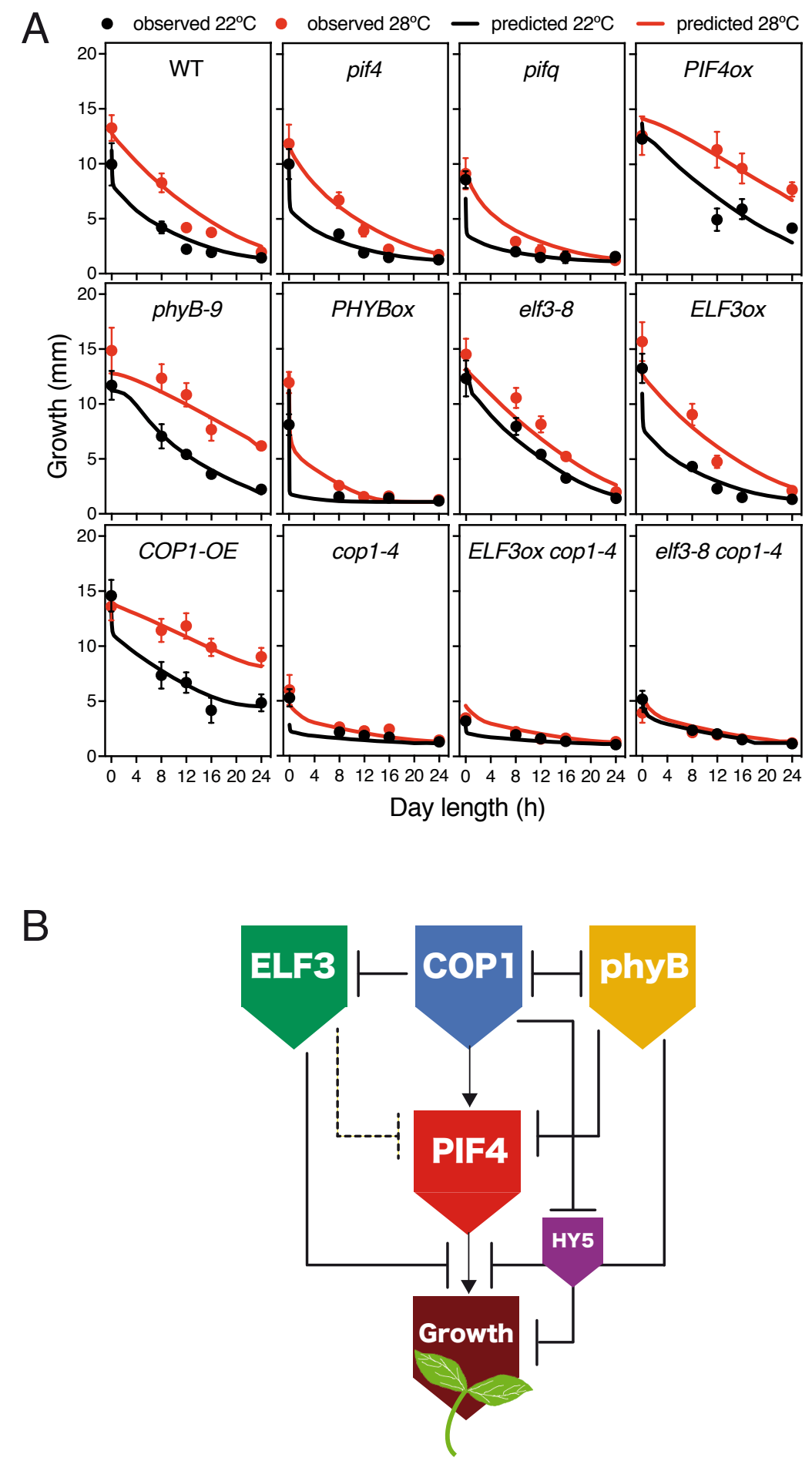

Figure 1. The phenotype of loss-of function mutants and overexpressors under different temperatures and day lengths.

(A) Growth of twelve Arabidopsis genotypes at either $22^{\circ} \mathrm{C}$ or $28^{\circ} \mathrm{C}$ under six white light daylength conditions. Observed measurements of hypocotyl length (circles) were compared with values predicted by the growth model (solid lines). Bars indicate standard deviation $(n=30)$. Despite its simplicity, the model accurately captures the main trends in the data. (B) Schematic representation of the network tested. Dashed and solid lines indicate transcriptional and post transcriptional regulations respectively . 


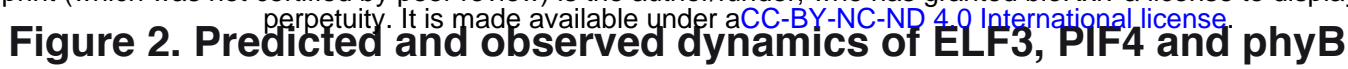
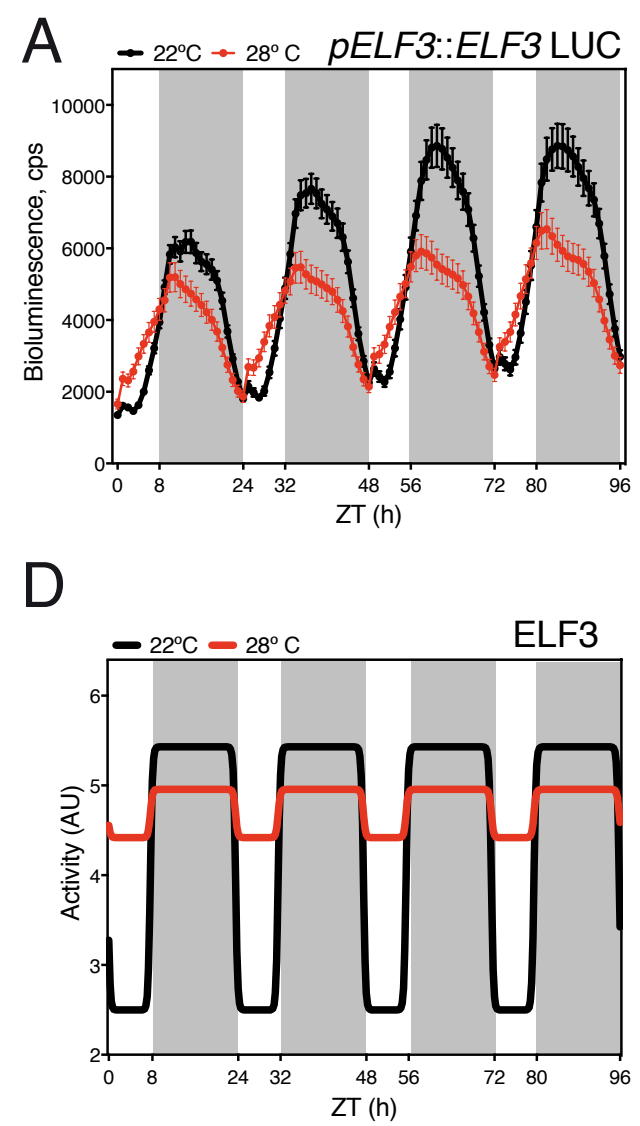

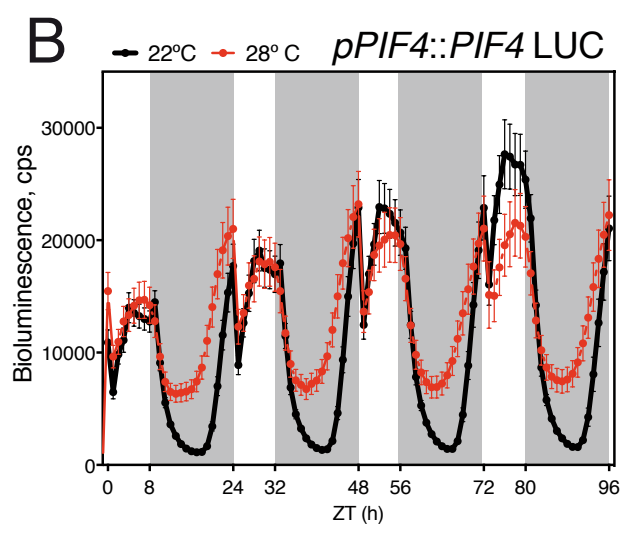

E

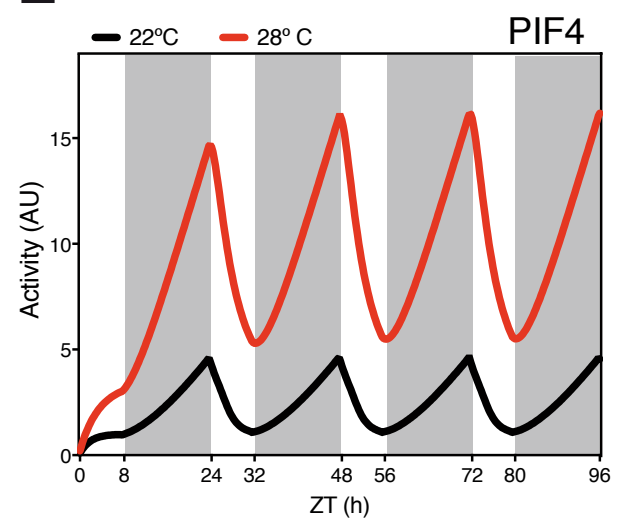

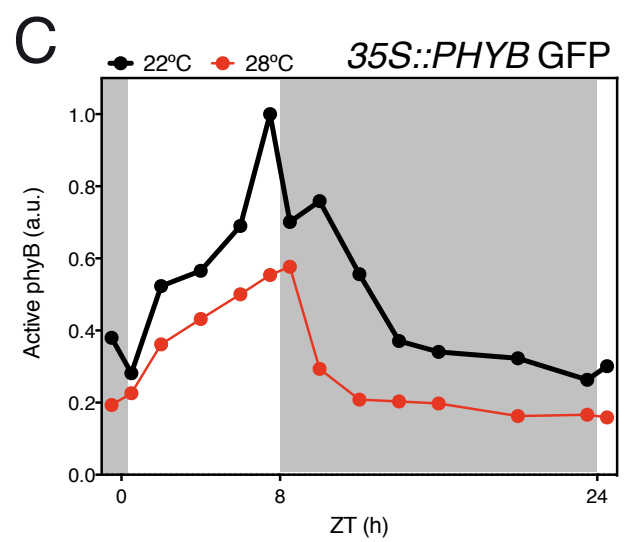

$\mathrm{F}$

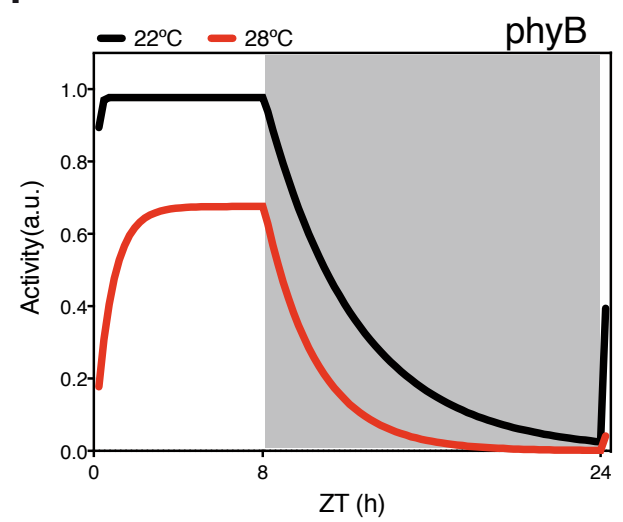

G

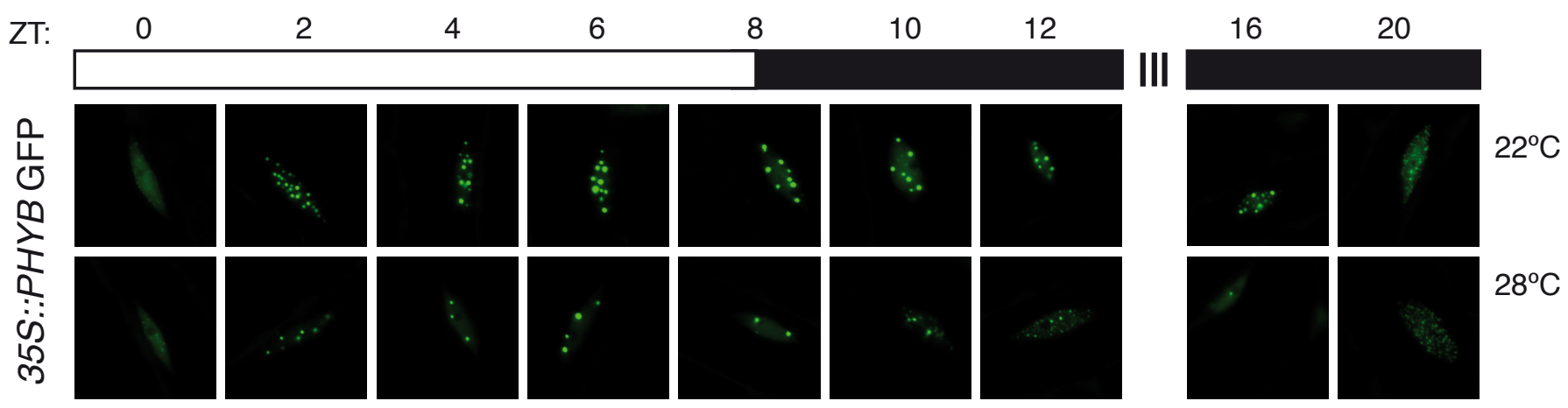

Figure 2. Predicted and observed dynamics of ELF3, PIF4 and phyB.

Bioluminescence assays of Col-0 lines expressing the construct pELF3::ELF3-LUC (A) and pPIF4::PIF4-LUC (B). Seedlings were grown in short day cycles at the indicated temperatures. Values shown represent mean +/- SEM of absolute bioluminescence of at least 24 seedlings. ZT indicates the zeitgeber time. Bioluminescence of seedlings was analyzed every hour. (D) ELF3 and (E) PIF4 activity predicted by the model. (C and G) phyB activity is affected by temperature in short day conditions. phyB-GFP transgenic seedlings were grown in short day cycles at either $22^{\circ} \mathrm{C}$ or $28^{\circ} \mathrm{C}$ for 5 days. Mean size of phyB nuclear bodies and total nuclear fluorescence was measured with Matlab Software (C) from confocal images and diagram of sampling time points and growth conditions (G). Arbitrary units (a.u.) of phyB activity were calculated by multiplying the mean size by nuclear fluorescence at each sampling time point. Ten seedlings were measured in each assay and two biological replicates were analyzed. Less photo bodies number and nuclear intensity correspond with warm temperature. All seedlings were grown under $50 \mu \mathrm{mol} . \mathrm{m}-2 . \mathrm{s}-1$ white light in short day cycles at $22^{\circ} \mathrm{C}$ or $28^{\circ} \mathrm{C}$. (F) phyB activity predicted by the model. The rectangles indicate the light conditions: white, lights on and grey, lights off. ZT indicates zeitgeber time. 
A
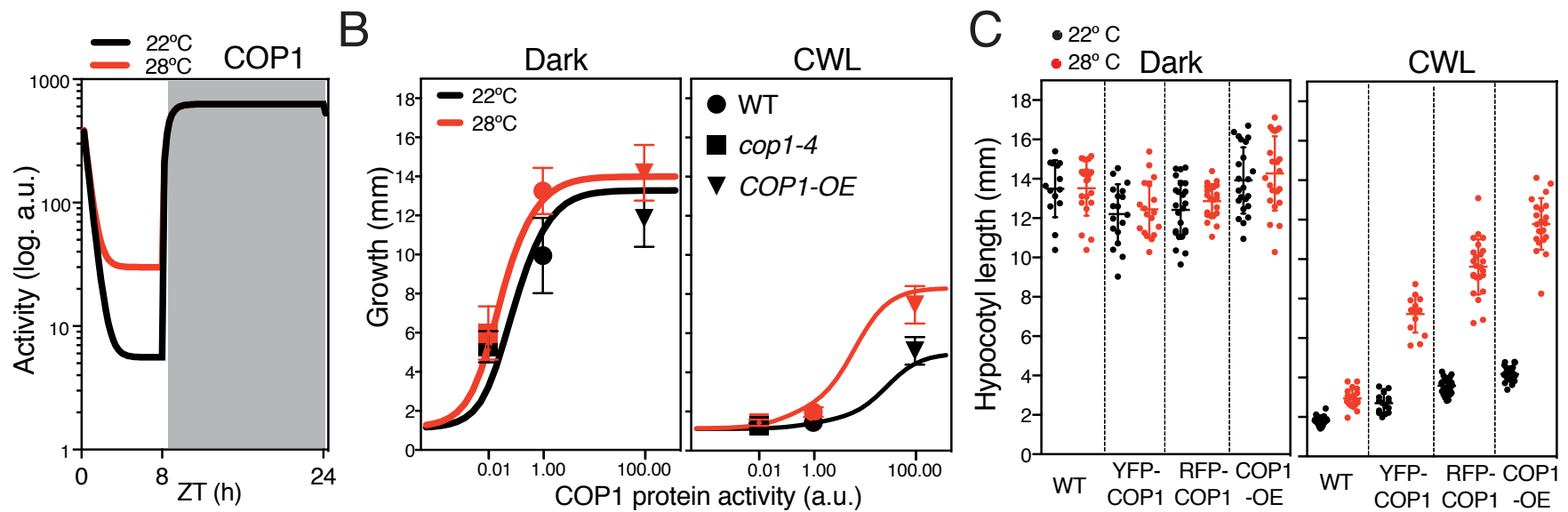

D
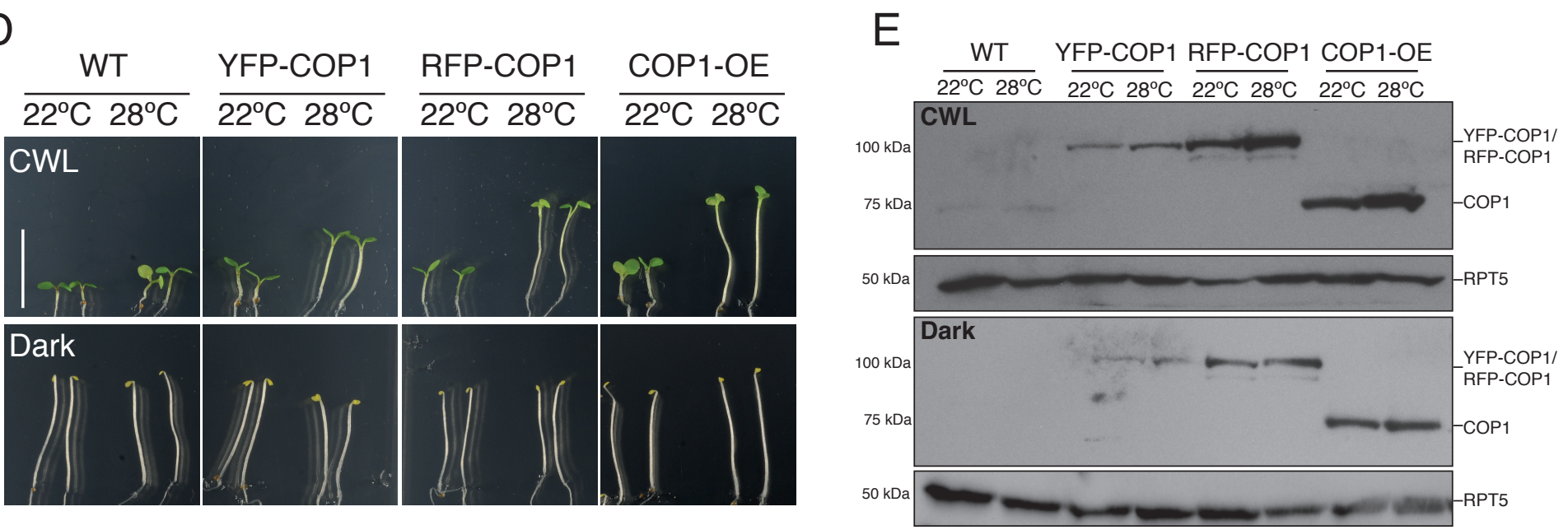

$\mathrm{F}$

G
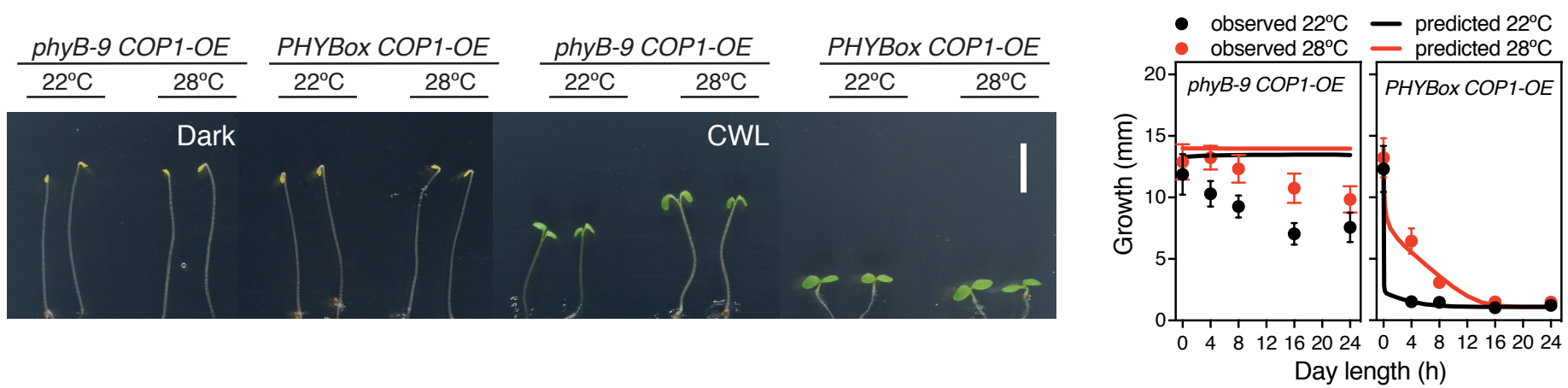

Figure 3. Predicted and observed dynamics of COP1

(A) COP1 activity levels predicted by the mathematical model in short day conditions. (B) Interaction of environmental conditions and COP1 levels. Observed measurements of Col-0, cop1-4 and COP1-OE hypocotyl length (symbols) versus the estimated values predicted by the model (solid lines) in dark (left panel) and CWL (right panel). (C) Graphs showing the hypocotyl length phenotypes. Bars indicate standard deviation $(n=30)$. (D) Pictures of five days old seedlings grown in CWL (upper panel) and continuous darkness (lower panel) at $22^{\circ} \mathrm{C}$ or $28^{\circ} \mathrm{C}$. Bar $=10 \mathrm{~mm}$. (E) Western blot showing that increased accumulation of overexpressed COP1 protein correlates with hypocotyl elongation in seedlings grown in CWL and saturated response in the darkness. COP1 was detected using an anti-COP1 antibody. RPT5 was used for loading control and was detected using an anti-RPT5 antibody. Total protein extracts were used for immunoblot analysis. (F) Phenotypes of seedlings of phyB-9 COP1-OE and $P H Y B o x$ COP1-OE grown for five days either in darkness or $\mathrm{CWL}$ at $22^{\circ} \mathrm{C}$ or $28^{\circ} \mathrm{C}$. (G) Observed values of hypocotyl growth of phyB-9 COP1-OE and PHYBOx COP1-OE (circles) versus the estimated values predicted by the model (solid lines) in dark (left panel) and CWL (right panel). 
bioRxiv preprint doi: https://doi org/10.1101/2021.1125.470009 - this version posted November 25, 2021. The copyright holder for this preprint (which was not certified by peer review) is the author/funder, who has granted bioRxiv a license to display the preprint in perpetuity. It is made available under aCC-BY-NC-ND 4.0 International license.

Figure 4. phyB, COP1 and ELF3 contribution to day length-dependent hypocotyl thermoelongation as predicted by the model

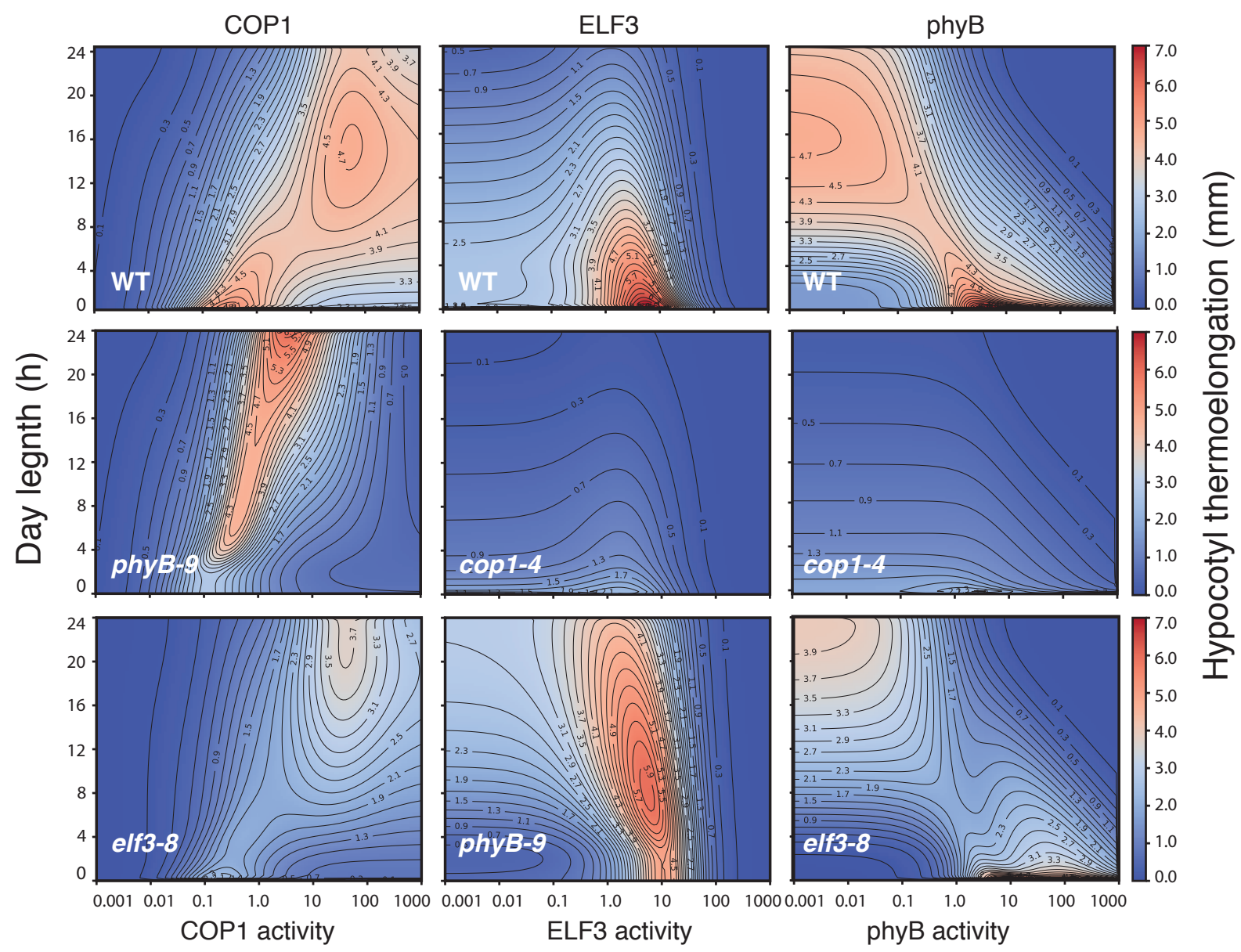

Figure 4. phyB, COP1 and ELF3 contribution to day length-dependent hypocotyl thermoelongation as predicted by the model.

Heatmap plots representing hypocotyl thermoelongation (calculated as the difference of hypocotyl length at $28^{\circ} \mathrm{C}$ and $22^{\circ} \mathrm{C}$ ) relative to day length ( $\mathrm{Y}$ axis) and activity of the COP1, phyB and ELF3 proteins ( $\mathrm{X}$ axis, as indicated). A value $=1.0$ corresponds to wild type level, while greater and lesser values are equivalent to overexpression and lossof-function, respectively. Backgrounds are indicated in the panels. 